\title{
Obtención de espumas metálicas de aluminio por el método de infiltración en preformas lixiviables.
}

\section{Obtaining Aluminum Metal foams by the method of infiltration into leachable} preform.

Patricio Abarca Pérez. ${ }^{1}$, Marcelo Castillo Cárdenas. ${ }^{2}$, David Bravo Morocho. ${ }^{3}$ \& Fabián Sánchez Carrión. ${ }^{4}$

Recibido: 01-12-2017 / Revisado:05-02-2018 Aceptado: 05-03-2018/ Publicado: 01-04-2018

\begin{abstract}
.
DOI: https://doi.org/10.33262/cienciadigital.v2i2.104

The objective of this study is to describe and analyze in a substantial way the method of obtaining metal foams from open cell aluminum, by means of the infiltration of molten metal method in leachable preforms, for which a significant number of samples were obtained. The preform used is $\mathrm{NaCl}$, with a grain size of 0.85 to 1.7 $\mathrm{mm}$. The experimental procedure is based on the use of an airtight cylindrical mold in which the aluminum infiltration in the preform takes place. A vacuum pressure is transmitted to this mold that allows to verify the suitable seal to be able to apply the positive pressure that favors the infiltration. The open cell metallic foams obtained have an average relative density of 0.145 , resulting in a high degree of porosity $(85.5 \%)$. This could be due to the wide range of particle size used, which denotes that a certain number of pores would have ligaments and cell walls thinner than others, therefore, require less metal.
\end{abstract}

Keywords: Metal Foam, Infiltration, Leach, Preform, Relative Density.

\section{Resumen.}

\footnotetext{
${ }^{1}$ Escuela Superior Politécnica de Chimborazo, Chimborazo, Ecuador, edison.abarca@espoch.edu.ec

${ }^{2}$ Escuela Superior Politécnica de Chimborazo, Chimborazo, Ecuador, edison.castillo@espoch.edu.ec

${ }^{3}$ Escuela Superior Politécnica de Chimborazo, Chimborazo, Ecuador, victor.bravo@espoch.edu.ec

${ }^{4}$ Escuela Superior Politécnica de Chimborazo, Chimborazo, Ecuador, esanchez_c@espoch.edu.ec
} 
El objetivo de este estudio es describir y analizar de una manera sustancial el método de obtención de espumas metálicas de aluminio de celda abierta, mediante el método infiltración de metal fundido en preformas lixiviables, para lo cual se obtuvieron un número significativo de muestras. La preforma utilizada es el $\mathrm{NaCl}$, con un tamaño de grano de 0,85 a $1,7 \mathrm{~mm}$. El procedimiento experimental se fundamenta en la utilización de un molde cilíndrico hermético en cuyo interior se produce la infiltración del aluminio en la preforma. A este molde se le transmite una presión de vacío que permite verificar el sello apto para poder aplicar la presión positiva que favorece la infiltración. Las espumas metálicas de celda abierta obtenidas, presentan una densidad relativa promedio de 0,145 , resultando en un alto grado de porosidad $(85,5 \%)$. Esto podría deberse al amplio rango de tamaño de partículas utilizado, lo cual denota que una cierta cantidad de poros tendrían ligamentos y paredes celulares más finas que otros, por tanto, requieren menos cantidad de metal.

Palabras Claves: densidad relativa, espuma metálica, infiltración, lixiviar, preforma

\section{Introducción.}

Las espumas metálicas constan dentro del grupo de materiales de nueva generación, son un caso especial de los sólidos celulares (Báez, Hernández \& Palomar, 2014). Se conocen dos tipos de espuma metálica, de celda cerrada (espuma) y celda abierta (esponja) (Wang et al., 2005). Se denominan de celdas abiertas si éstas se conectan entre sí, y celdas cerradas si sucede lo contrario (Medik et al., 2017).

Las espumas metálicas exhiben favorables propiedades mecánicas y físicas, tales como elevada rigidez, resistencia al impacto, y alta conductividad térmica, todo esto combinado con su baja densidad, lo cual posibilita una serie de aplicaciones (Medik et al., 2017), entre las cuales se destacan estructuras ligeras, intercambiadores de calor, elementos de filtro, absorbentes acústicos, elementos de rigidez, amortiguadores, compuestos de matriz metálica, entre otras (Wang et al., 2005).

Las espumas metálicas presentan propiedades excepcionales si se las compara a las propiedades del mismo metal, pero sin poros (Michailidis, Stergioudi \& Tsipas, 2010), dichas propiedades están directamente relacionadas con su densidad relativa y estructura del material (Michailidis et al., 2010).

Ciertos rasgos macroscópicos de su estructura, tales como, tamaño de poro, tipo de celda, espesor de pared celular y ligamentos, curvatura de paredes de celda, entre otras, marcan una trascendental influencia sobre su comportamiento mecánico, y pueden ser parcialmente 
controlados a través de procedimientos y/o técnicas que generalmente resultan costosas y complejas (Gutiérrez \& Oñoro, 2008)

La investigación acerca de las espumas metálicas requiere ahondar en temas referentes a la refinación de los procesos para su obtención o fabricación, lo cual implica el abaratamiento de costos y acceder a un control apropiado sobre su estructura y morfología. Además es sustancial aportar con mayor información confiable, con respecto a la relación que existe entre las propiedades mecánicas de las espumas y su morfología, su estructura, entre otras (Irausquin, 2012).

\section{Importancia del problema.}

Una de las técnicas principalmente desarrolladas para obtener espumas metálicas de celda abierta se basa en utilizar preformas removibles, de las cuales es de especial interés el método de infiltración del metal fundido en preformas solubles (Nebreda, 2014). Este método destaca por su relativa simplicidad experimental, y porque ofrece un importante grado de control sobre la estructura de la espuma (Banhart, 2000).

En lo que se refiere a materiales y equipos utilizados, este método resulta práctico, sencillo y económico (Roldán \& Ángel, 2012). En la bibliografía disponible existen varios trabajos referentes a este tema, de los cuales muchos de ellos, o son demasiado resumidos, o carecen de algunas especificaciones muy importantes, como por ejemplo, la estimación de la presión necesaria para una adecuada infiltración, entre otros.

Por lo tanto, en este artículo se presenta una descripción fundamental del procedimiento y de los principales parámetros que involucra el método de infiltración para obtener espumas metálicas de aluminio de celda abierta, para de esta manera ser un aporte y una referencia que permita desarrollar este proceso in situ.

\section{Metodología.}

\section{Equipos y materiales utilizados.}

El proceso a ser descrito se basa en la infiltración de aluminio fundido alrededor de una preforma de partículas de $\mathrm{NaCl}$, estas partículas se disuelven mediante agua y por ende definen la porosidad final del material (Luna et al., 2014). El esquema de los equipos y materiales utilizados se muestran en la Figura 1. 
Figura 1. Esquema de los equipos y materiales utilizados para obtener espumas metálicas de celda abierta.

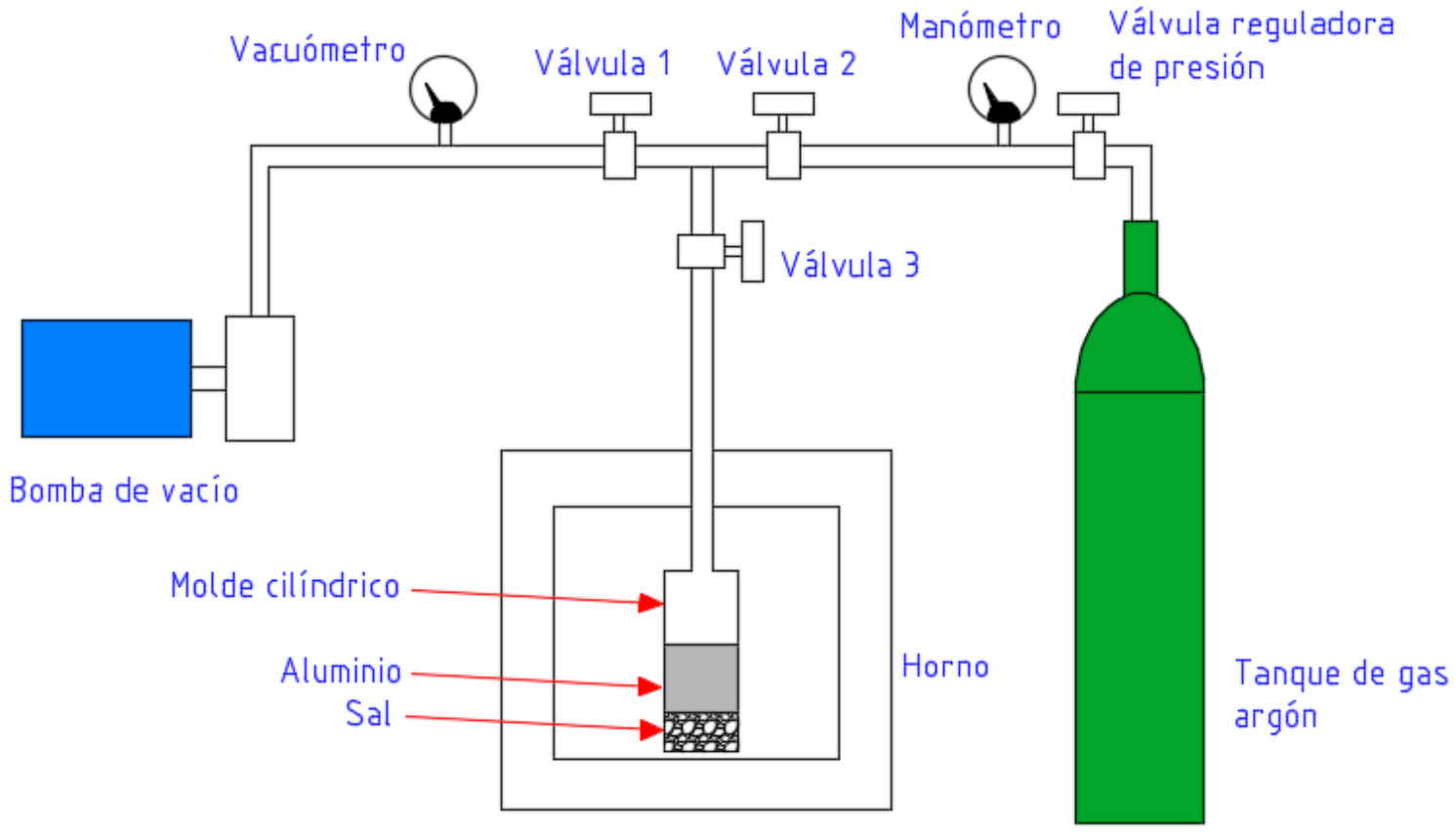

Fuente: (Abarca, 2017).

\section{Material para la preforma.}

Es muy importante que la preforma no se funda durante el proceso. La preforma de $\mathrm{NaCl}$ (p.f. $801{ }^{\circ} \mathrm{C}$ ) es ideal para producir espumas con metales cuyo punto de fusión estén por debajo a $750^{\circ} \mathrm{C}$, por tanto, el aluminio (p.f. $660{ }^{\circ} \mathrm{C}$ ) se ajusta perfectamente. $\mathrm{El} \mathrm{NaCl}$ es químicamente inerte en contacto con el aluminio (fundido o sólido) lo cual es requerido durante el proceso (Conde et al., 2006). $\mathrm{El} \mathrm{NaCl}$ se lixivia mediante agua, y no ocasiona ninguna amenaza considerable para la salud y medio ambiente, además es poco costoso (Laughlin \& Hono, 2014). El tamaño de poro de la espuma será una réplica del tamaño de grano de $\mathrm{NaCl}$ removido, por tanto, se seleccionan los tamices apropiados, para este caso se opta por un tamaño de grano en el rango de 0,85 a $1,7 \mathrm{~mm}$.

Al conocer la geometría del molde y el tamaño de grano de $\mathrm{NaCl}$, se puede variar la cantidad de $\mathrm{NaCl}$ a usar para infiltración dependiendo de la altura de espuma requerida, entre 100g y 300g, para este caso se emplea $200 \mathrm{~g}$ (Abarca, 2017). 
2. Fabricación de barras de aluminio que sirven para obtener los tochos de aluminio para la infiltración.

Las barras cilíndricas de aluminio se obtuvieron mediante el proceso de fundición en molde cerrado de arena (Groover, 2006). Las barras producidas, deben tener un diámetro de 50 $\mathrm{mm}$, para lo cual la cavidad interna cilíndrica del molde de arena se calcula de tal manera que se contrarreste los efectos de contracción del metal durante la solidificación, para este caso dio buenos resultados con un diámetro de $52 \mathrm{~mm}$ (Abarca, 2017).

\section{Preparación de los tochos de aluminio para la infiltración.}

Las barras de aluminio deben ser mecanizadas de tal manera que alcancen el diámetro deseado $(50 \mathrm{~mm})$ y además adquieran un adecuado acabado superficial (Abarca, 2017). Posteriormente estas barras son seccionadas en piezas de 3 o $4 \mathrm{~cm}$ de longitud, dependiendo de la cantidad de $\mathrm{NaCl}$ a ser infiltrado.

\section{Fabrica del molde cilíndrico.}

El material seleccionado para fabricar el molde cilíndrico es el acero inoxidable AISI 304, puesto que su punto de fusión $\left(1454{ }^{\circ} \mathrm{C}\right)$ está muy por encima de la temperatura del proceso, y además tiene una buena resistencia a la corrosión (Luna et al., 2014). El molde está compuesto por un tubo cilíndrico $(60 \times 8 \times 150 \mathrm{~mm})$ que se acopla mediante cuatro varillas roscadas a dos placas (100x100x10mm) ranuradas superior e inferior (Figura 2).

Las ranuras son circulares y concéntricas en las placas, sirven tanto para acoplarse al cilindro como para alojar los anillos de grafito que permitirán sellar y hermetizar el cilindro durante el experimento (Luna et al., 2014). La placa superior posee un conducto (Figura 2c) que permite conectarse a un sistema de válvulas, el cual posibilita transmitir al cilindro una presión negativa y positiva respectivamente. La presión negativa es requerida para verificar el sello entre las placas y el cilindro del molde. La presión positiva favorece que el aluminio se infiltre en los espacios intersticiales de la preforma (Abarca, 2017).

\section{Horno mufla eléctrico utilizado en el proceso.}

Para el proceso de infiltración se utiliza un horno mufla eléctrico con control digital puesto que permite un alto grado de control de la temperatura. El interior del molde debe alcanzar $740{ }^{\circ} \mathrm{C}$ aproximadamente, que es lo recomendado para el proceso (Luna et al., 2014). Si el procedimiento se lleva a cabo a una temperatura menor, el aluminio podría no infiltrarse (Abarca, 2017).

\section{Bomba de vacío.}


Es importante crear un vacío al interior del molde con el fin de verificar el sello y la hermeticidad que posee el molde.

\section{Tanque de gas argon a presión.}

Para generar la presión positiva que facilitará la infiltración del aluminio dentro del molde, se utiliza gas argón (Abarca, 2017). El argón al ser un gas noble, es idóneo para el proceso porque no reacciona con ningún otro elemento.

El oxígeno es altamente activo en superficie de muchos metales fundidos, y su presencia a niveles relativamente bajos puede producir una disminución sustancial de la tensión superficial (Keene, 1993), lo cual favorecería la infiltración. La tensión superficial del aluminio fundido expuesto a condiciones normales de ambiente se presenta en la Figura 2.

Figura 2. Tensión superficial del aluminio puro.

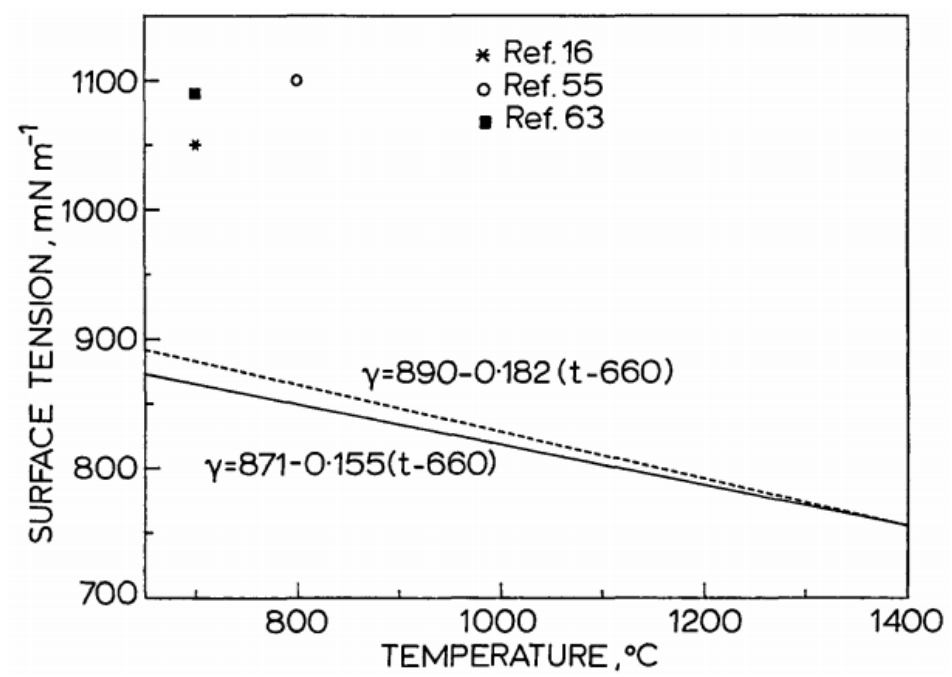

Fuente: (Keene, 1993).

Una relación matemática útil para poder estimar la presión de infiltración en preformas está dada por (Aguilar, 2008):

$$
\Delta P=\frac{6 * \lambda * \gamma_{l v} * \cos \theta *(1-w)}{D * w}
$$

Dónde:

$\Delta P=$ Presión crítica para iniciar la infiltración

$D=$ Diámetro promedio de las partículas

$w=$ Fracción de poros en la preforma

$\lambda=$ Factor geométrico (usualmente igual a 1,4)

EDUCACIÓN $\gamma_{l v}=$ tensión superficial del líquido

$\theta=$ ángulo de contacto 
Tomando en cuenta que la infiltración del aluminio se llevará a $740{ }^{\circ} \mathrm{C}\left(\gamma=0,846 \frac{\boldsymbol{N}}{m}\right)$, además que el diámetro promedio de las partículas más pequeñas de los granos de $\mathrm{NaCl}$ es de $0,85 \mathrm{~mm}$, y que la porosidad obtenida de las espumas es de 0,85 (Tabla 1), la presión de infiltración estimada es:

$\Delta P=\frac{6 * 1,4 * 0,846 \frac{N}{m} * \cos 45 *(1-0.85)}{(0,00085 m) * 0,85}=9835,81 \frac{N}{m^{2}} \approx 0,1$ bar

Este valor indica que, para una adecuada infiltración, el gas argón debería ejercer una presión mayor a 0,1 bar.

\section{Anillos de grafito.}

A partir de una lámina de grafito de $2 \mathrm{~mm}$ de espesor, se obtienen dos anillos de grafito (DE=60mm y $\mathrm{DI}=51 \mathrm{~mm}$ ), los mismos deberán calzar perfectamente dentro de las ranuras de las placas del molde cilíndrico respectivamente. Estos anillos posibilitan que el molde cilíndrico permanezca hermético durante el experimento.

\section{Sistema de válvulas.}

Se hará uso de un sistema compuesto por tres válvulas: la válvula 1 conecta o desconecta la bomba de vacío con el molde, la válvula 2 conecta o desconecta el tanque de gas argón con el molde, y la válvula 3 conecta o desconecta el molde del resto del sistema (Figura 1).

Procedimiento para obtener espuma metálica de celda abierta (Luna et al., 2014) (Abarca, 2017):

1. El procedimiento de preparación del molde cilíndrico listo para la infiltración se muestra en la Figura 3.

2. Acoplar el tubo que se deriva de la placa superior del molde al sistema de válvulas. Cerrar todas las válvulas.

3. Abrir las válvulas que conectan la bomba de vacío con el molde (válvula 3 y válvula 1) (Figura 1). Poner en marcha la bomba hasta que el vacuómetro indique la menor presión posible, y apagarla. Si la pérdida de vacío en el sistema es inferior a 0,0666 Bar/s para los primeros 10 segundos después de apagar la bomba, el sello en el molde es adecuado para la infiltración.

4. Dejar la válvula 3 abierta y cerrar la válvula 1. Sin desmontar el sistema de válvulas, 
ubicar el molde dentro del horno precalentado y esperar por $1 \mathrm{~h}$.

5. Cerrar todas las válvulas del sistema. Abrir la válvula 2, la cual conduce al cilindro de gas argón, seguidamente abrir la válvula principal del tanque de gas argón, y fijar una presión de infiltración mayor a 0,1 bar. Rápidamente, abrir la válvula 3 .

6. Después de aproximadamente $1 \mathrm{~min}$, extraer el molde del horno, y para acelerar la perdida de calor, ubicarlo sobre una superficie metálica.

Figura 3. (a) Placa inferior del molde sobre las varillas roscadas. (b) Anillo de grafito dentro de la ranura de la placa inferior. (c) Cámara cilíndrica dentro de la ranura de la placa inferior. (d) Granos de $\mathrm{NaCl}$ dentro del molde cilíndrico. (e) Tocho de aluminio dentro del molde. (f) Anillo de grafito dentro de la ranura de la placa superior. (g) Molde cilíndrico ensamblado completamente.

a)

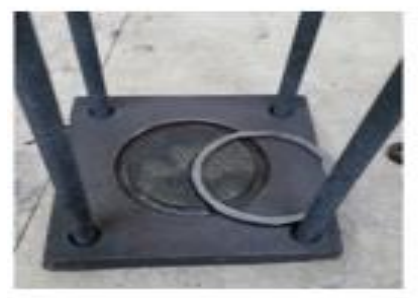

b)

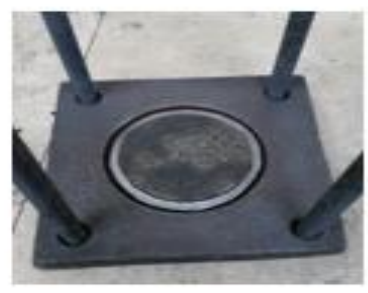

d)

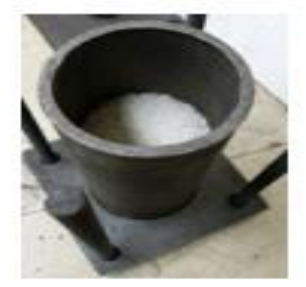

f)

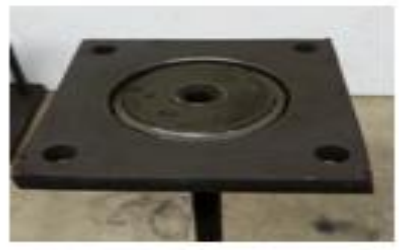

c)

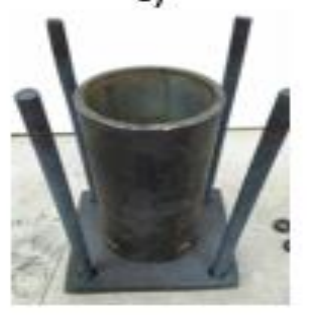

e)
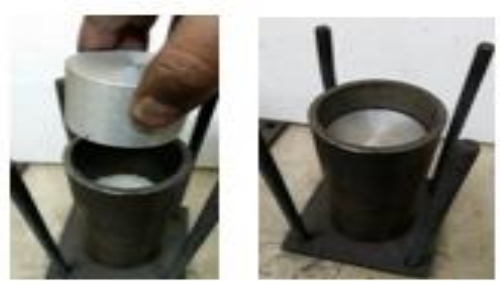

g)

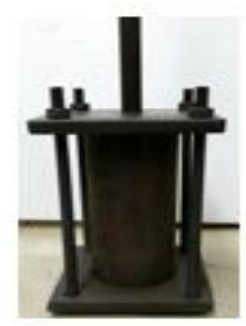

Elaborado por: Grupo de Investigación.

7. Cuando el molde haya disminuido su temperatura, desconectar el sistema de válvulas, desmontar las placas del cilindro. Seguidamente extraer la muestra de espuma infiltrada obtenida y ubicarla en un recipiente con agua expuesto a la llama, esperar hasta disolver completamente la preforma de $\mathrm{NaCl}$. 
8. Mediante una sierra de banda remover el excedente de aluminio no infiltrado en la preforma de $\mathrm{NaCl}$.

\section{Análisis de Resultados.}

Mediante el proceso descrito, se pudo obtener un número representativo de muestras de espumas (Figura 4). Se determino su densidad relativa, la cual es considerada uno de los parámetros más importantes, además se calculó la porosidad y el grado de expansión del metal base (Tabla 1). Estos parámetros se definen como (Nebreda, 2014):

$$
\begin{gathered}
\rho_{r}=\frac{\rho}{\rho_{s}} \\
p \approx 1-\frac{\rho}{\rho_{s}}=1-\rho_{r} \\
G=\frac{V}{V_{s}}=\frac{\rho_{s}}{\rho}=\frac{1}{\rho_{r}}
\end{gathered}
$$

Dónde: $\quad \rho_{r}=$ densidad relativa

$$
\begin{aligned}
& \rho=\text { densidad del material celular } \\
& \rho_{s}=\text { densidad del material base } \\
& p=\text { porosidad }
\end{aligned}
$$

Figura 4. Muestra de espuma metálica de celda abierta obtenida: a) Muestra sin remover el aluminio no infiltrado. b) Muestra libre del aluminio no infiltrado.
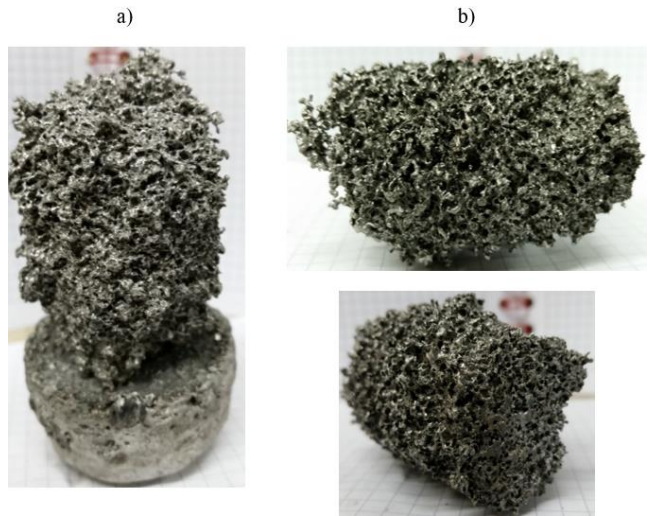

Elaborado por: Grupo de Investigación. 
Tabla 1. Datos de densidad relativa, porosidad y grado de expansión de tres muestras de espuma metálica obtenidas por el método de infiltración.

\begin{tabular}{|c|c|c|c|c|c|c|}
\hline Muestra & $\begin{array}{lr}\text { Tamaño } & \text { de } \\
\text { partícula }(\mathrm{mm}) \\
\text { de NaCl usado } \\
\text { como preforma }\end{array}$ & $\begin{array}{l}\text { Densidad } \\
\text { relativa }\end{array}$ & Porosidad & $\begin{array}{l}\% \\
\text { Porosidad }\end{array}$ & $\begin{array}{l}\text { Grado de } \\
\text { expansión }\end{array}$ & $\begin{array}{l}\text { \% Grado } \\
\text { de } \\
\text { expansión }\end{array}$ \\
\hline 1 & $0,85-1,7$ & 0,138 & 0,862 & 86,2 & 7,246 & 724,638 \\
\hline 2 & $0,85-1,7$ & 0,171 & 0,829 & 82,9 & 5,848 & 584,795 \\
\hline \multirow[t]{3}{*}{3} & $0,85-1,7$ & 0,154 & 0,846 & 84,6 & 6,494 & 649,351 \\
\hline & Media & 0,154 & Media & 84,567 & Media & 652,928 \\
\hline & $\begin{array}{l}\text { Desviación } \\
\text { estándar }\end{array}$ & 0,017 & $\begin{array}{l}\text { Desviación } \\
\text { estándar }\end{array}$ & 1,65 & $\begin{array}{l}\text { Desviación } \\
\text { estándar }\end{array}$ & 69,99 \\
\hline
\end{tabular}

Elaborado por: Grupo de Investigación.

Los resultados muestran que las espumas metálicas obtenidas por infiltración, en las cuales se utilizó un tamaño de partículas de $\mathrm{NaCl}$ de $0.85-1.7 \mathrm{~mm}$, tienen un valor medio de porosidad de $85.5 \%$. Según reportes de otra investigación, para un tamaño de partículas de $\mathrm{NaCl}$ en el rango de $1.4-1.7 \mathrm{~mm}$, la porosidad registrada tiene un valor promedio de $63.2 \%$ (Luna et al., 2014).

Se debe tomar en cuenta de que a menor tamaño de partículas de $\mathrm{NaCl}$, se crearán poros más estrechamente espaciados, y como consecuencia sus paredes celulares y ligamentos serán más finos (Hussain \& Suffin, 2011).

Gracias a esta información la variación observada de porosidad, podría deberse a que para la presente investigación se utilizó un rango de partículas más amplio, es decir, se tuvo una combinación de partículas más finas $(0,85-1,7 \mathrm{~mm})$, por tanto, una cierta cantidad de poros tendrán ligamentos y paredes celulares más finos que otros, de esta manera se requiere menos cantidad de metal, lo cual dará como resultado una menor densidad relativa, y por ende una mayor porosidad.

\section{Conclusiones.}

- Las espumas metálicas de celda abierta obtenidas por el método de infiltración, presentan una densidad relativa promedio de 0,154 , esto significa un alto grado de porosidad (84,6\%). Esto podría deberse al amplio rango de tamaño de partículas utilizado $(0.85$ - $1.7 \mathrm{~mm})$, lo que significa que una cierta cantidad de poros tendrán ligamentos y paredes celulares más finos que otros, de esta manera se requiere menos cantidad de metal. La influencia del aire dentro del molde durante el proceso, 
no perjudica de ninguna manera la infiltración del aluminio a través de las partículas de $\mathrm{NaCl}$, más bien la reacción con el oxígeno disminuye la tensión superficial del aluminio líquido, lo cual favorece la infiltración.

- Es importante controlar adecuadamente la temperatura del proceso, puesto que, si se emplea temperaturas relativamente bajas, el aluminio no se funde y por tanto no existirá infiltración. En cambio, si las temperaturas son demasiado altas, se corre un gran riesgo de fundir a las partículas de sal con lo cual afectaría enormemente la estructura final de la espuma.

\section{Referencias bibliográficas.}

Báez, S., Hernández, M., y Palomar, M. (2014). Processing and characterization of opencell aluminum

foams obtained through infiltration processes, Science Direct, 44, 54-61.

Wang, L., Li, H., Wang, F., y Ren, J. (2005). Preparation of open-cell metal foams by investment cast,

China Foundry, 2 (1), 56-59.

Medik, F., Sunar, T., Cetik, M., Yasar, M., y Turban, L. (2017). Production of Open Cell Aluminum Foam

via Infiltration Method, 1st International Conference of Advanced Materials and Manufacturing Technologies

(ICAMT'17), pp. 101-104.

Michailidis, N., Stergioudi, F., y Tsipas, D. (2010). Manufacturing of Open-Cell Metal Foams Using a

Novel Leachable Pattern, Advanced Engineering Materials, 13 (1-2), 29-32.

Nebreda, J. (2014). Optimización de la estructura celular en espumas de aluminio (Tesis Doctoral).

Universidad de Valladolid, Valladolit, España.

Banhart, J. (2001). Manufacture, characterisation and application of cellular metals and metal foams,

Progress in Materials Science, 46 (6), 559-632.

Roldán, D., y Ángel, B. (2012). Análisis del costo de producción de Esponjas de aluminio. II Encuentro de

Investigación Formativa, Medellín, Colombia: Editorial Universidad Pontificia Bolivariana.

Conde, Y., Despois, F., Goodall, R., Marmottant, A., Salvo, L., San Marchi, C., y Mortensen, A. (2006).

Replication Processing of Highly Porous Materials, Advanced Engineering Materials, 8 (9), 795-803. 
Elizondo, E., Barari, F., Woolley, R., y Goodall, R. (2014). Casting Protocols for the Production of Open

Cell Aluminum Foams by the Replication Technique and the Effect on Porosity, Journal of Vizualized

Experiments.

Abarca, P. (2017). Síntesis de espumas metálicas de celda abierta (Tesis de Maestría).

Escuela Politécnica

Nacional, Quito, Ecuador.

Laughlin, D., y Hono, K.. (2014). Physical Metallurgy. Lausanne, Switzerland: Elsevier.

Groover, M. (2007). Fundamentos de manufactura moderna. México DF, México: McGraw-Hill Interamericana.

Hussain, Z., y Suffin, N. (2011). Microstructure and Mechanical Behaviour of Aluminium Foam Produced

by Sintering Dissolution Process Using $\mathrm{NaCl}$ Space Holder, Jornal of Enginering Science, 7, 37-49.

Keene, B., (1993). Review of data for the surface tension of pure metals, The Institute of Materials and ASM

International , 158-192.

Aguilar, J., y Hernández, B. (2008). La infiltracion no asistida como una técnica de procesamiento de

compositos Al-Mg-Si/SiC, Revista Mexicana de Física, 54 (5), 336-340.

Gutiérrez, J., y Oñoro, J. (2008). Espumas de aluminio.Fabricación,propiedades y aplicaciones. Revista de

Metalurgia, 44 (5), 457-476.

Irausquin, I. A. (2012). Caracterización mecánica de espumas metálicas y su aplicación en sistemas de

absorción de energía (Tesis Doctoral). Universidad Carlos III de Madrid, Madrid, España.

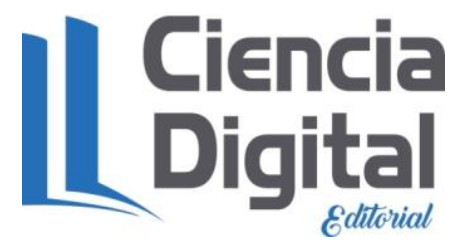


Para citar el artículo indexado.

Abarca P., Castillo M., Bravo D. \& Sánchez F. (2018). Obtención de espumas metálicas de aluminio por el método de infiltración en preformas lixiviables. Revista electrónica Ciencia Digital 2(2), 348-360. Recuperado desde: http://cienciadigital.org/revistacienciadigital2/index.php/CienciaDigital/article/view/105/98

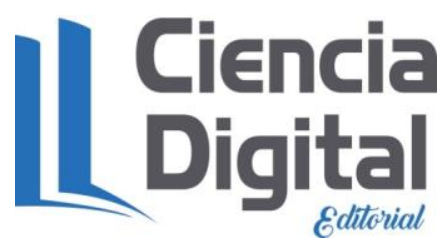

El artículo que se publica es de exclusiva responsabilidad de los autores y no necesariamente reflejan el pensamiento de la Revista Ciencia Digital.

articulo queda en propiedad de la revista y, por tanto, su publicación parcial y/o total en otro medio tiene que ser autorizado por el director de la Revista Ciencia Digital.
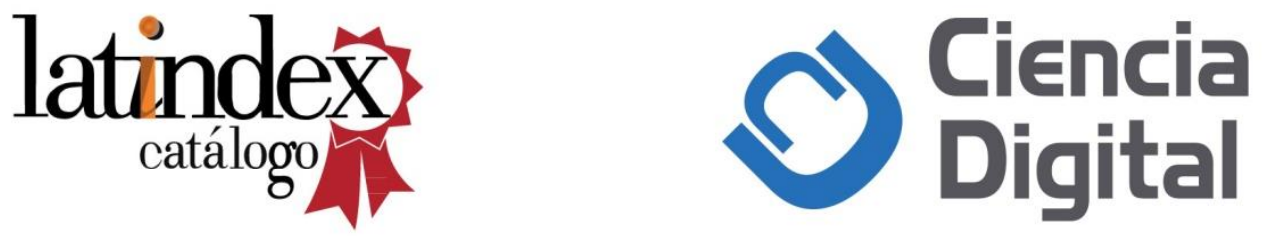